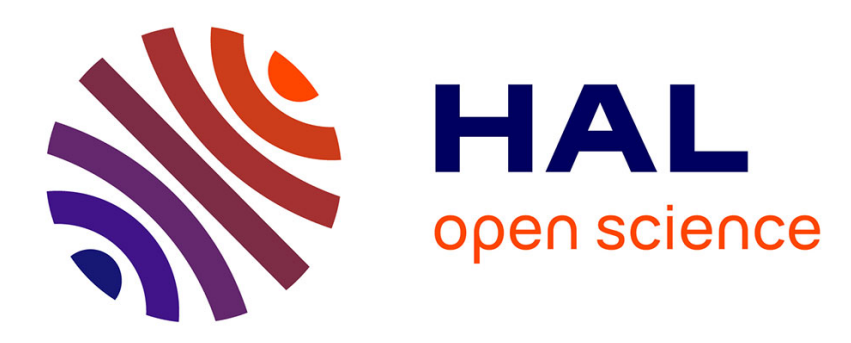

\title{
Productivité académique contre contribution scientifique: le cas de l'économie
}

\author{
Serge Walery
}

\section{To cite this version:}

Serge Walery. Productivité académique contre contribution scientifique: le cas de l'économie. L'Économie politique, 2011, 2011/3 (51), pp.70-96. 10.3917/leco.051.0070 . hal-01224429

\author{
HAL Id: hal-01224429 \\ https://hal.science/hal-01224429
}

Submitted on 5 Nov 2015

HAL is a multi-disciplinary open access archive for the deposit and dissemination of scientific research documents, whether they are published or not. The documents may come from teaching and research institutions in France or abroad, or from public or private research centers.
L'archive ouverte pluridisciplinaire HAL, est destinée au dépôt et à la diffusion de documents scientifiques de niveau recherche, publiés ou non, émanant des établissements d'enseignement et de recherche français ou étrangers, des laboratoires publics ou privés. 


\section{Productivité académique contre contribution scientifique : le cas de l'économie}

«Plus vous voudrez accélérer les progrès de la science, et plus vite vous la ferez périr. »

[Nietzsche, 1874]

Depuis quelques années, les conditions et modalités d'évaluation de la recherche scientifique ont connu de multiples et profondes transformations. L'harmonisation européenne, la multiplication des comparaisons et classements internationaux, le développement des techniques et des indicateurs bibliométriques, l'influence croissante des listes classant les revues, la systématisation des financements sur projets et/ou appels d'offres, la création en France de l'ANR ${ }^{1}$ puis de l'AERES ${ }^{2} \ldots$ sont autant d'éléments qui ont participé à ce bouleversement du paysage de l'évaluation de la recherche, et qui ont, chacun, donné lieu à d'innombrables réflexions, débats et contributions. Pour autant, ces analyses, aussi nécessaires et convaincantes soient-elles, se cantonnent généralement à l'étude d'un ou de quelques éléments de ce paysage.

On se propose ici, à l'inverse, de privilégier une appréhension globale de ces différents éléments, c'est-à-dire de souligner en quoi ils «font » système ou régime, et d'appréhender ce que tendent à devenir, dans le cadre de ce régime, le travail des chercheurs, les conditions de production de leurs travaux, et donc, la dynamique de la connaissance scientifique. Après une caractérisation, aussi synthétique que possible, du régime d'évaluation qu'on qualifiera de "quantitatif", on s'efforcera de montrer à quel point celui-ci incite au conformisme des chercheurs, et génère une standardisation du travail de recherche conduisant à l'asphyxie progressive de la dynamique de la connaissance scientifique. On remarquera, qu'en économie, cette tendance est sensiblement renforcée par la dérive instrumentaliste de la discipline.

La plupart des phénomènes qui seront évoqués touchent, à des degrés divers, l'ensemble des disciplines scientifiques. Les considérations qui suivent, centrées sur le cas de l'économie (et sur l'univers institutionnel français), ne sauraient cependant être étendues, sans précautions, aux autres sciences sociales, et encore moins aux sciences "dures".

\section{Le régime d'évaluation quantitative (REQ)}

\section{Dynamique et acteurs}

Dans le cadre de la tendance historique de longue durée au développement des appareils statistiques et au souci croissant d'efficacité de l'action publique [Desrosières 2010], l'émergence puis l'affirmation d'un "régime d'évaluation quantitative" (REQ) de la recherche, au cours des dernières décennies, résultent, concrètement, de la

\footnotetext{
${ }^{1}$ L'Agence Nationale de la Recherche, créée en février 2005 est une agence de moyens chargée de la mise en œuvre de la programmation scientifique, par l'attribution de financements sur appels d'offres et sur projets.

${ }^{2}$ Agence d'Évaluation de la Recherche et de l'Enseignement Supérieur, autorité administrative indépendante créée en mars 2007.
} 
combinaison/conjonction de trois dynamiques, chacune portée par une catégorie d'acteurs (managers, évaluateurs, chercheurs) du "monde" de la recherche.

Ce régime d'évaluation participe tout d'abord de la montée en puissance générale des préoccupations en matière d'évaluation des politiques publiques, et de l'évolution des moyens mis en œuvre pour y répondre [Perret 2006, 2008]. De l'époque de la rationalisation à celle de l'évaluation, puis à celle du "nouveau management public", la volonté politique d'un management plus efficace de la recherche a logiquement conduit à un renforcement du contrôle et des processus d'évaluation des institutions et des individus. Sur fond de compétition internationale et $\mathrm{d}$ 'harmonisation européenne, les récentes réformes institutionnelles (AERES, ANR, réforme du CNU...) et le poids croissant des financements (européens, nationaux ou locaux) sur projets, s'inscrivent clairement dans cette perspective.

$\mathrm{Du}$ point de vue des managers de la recherche, intimement convaincus de la « flânerie » des chercheurs comme Taylor l'était de celle des ouvriers, ${ }^{3}$ le développement et le renforcement des processus d'évaluation visent, par le renforcement de la compétition académique, statutaire et économique, à l'amélioration de la productivité (intensification) du travail des chercheurs et des laboratoires. Or, comme pour toute autre activité, l'affirmation de la logique manageriale dans le monde de la recherche s'accompagne nécessairement d'une obsession quantitativiste.

"Le souci de l'efficacité, assis sur la mesure de la performance, place l'activité de quantification au cour de l'action publique : sans chiffres, on ne peut ni fixer des objectifs, ni définir des indicateurs de performance, ni contrôler la productivité, ni annoncer des résultats, ni évaluer le degré de réussite d'une disposition de politique publique. » [Ogien, 2010]

La montée en puissance du REQ est également le fruit de la réalité matérielle à laquelle se trouvent confrontés les évaluateurs. Comme le souligne un récent rapport de l'Académie des Sciences, ${ }^{4}$ les modalités traditionnelles d'évaluation sont dévoreuses de temps. Dès lors, la prolifération des évaluations rend inéluctablement ces modalités obsolètes.

À tous les échelons, les instances d'évaluation sont concrètement dépassées par la masse des dossiers à évaluer. Qu'on songe à l'AERES, chargée d'évaluer, tous les quatre ans, et dans les disciplines les plus variées, les laboratoires, les formations, les universités, et, de fait, les 55000 enseignants-chercheurs qui les composent; à la $5^{\text {ème }}$ section (économie) du Conseil National des Universités (CNU-5), ${ }^{5}$ dont les 48 membres traitent, chaque année, 300 à 350 dossiers de candidats à la qualification des Maîtres de Conférences (MCF) ${ }^{6}$; ou aux Comités de sélection des universités, parfois confrontés à un nombre de candidats à trois chiffres, pour un poste de MCF. Dans de telles conditions, les membres de ces institutions, contraints de rationaliser leur travail d'évaluation, ${ }^{7}$ sont naturellement conduits à recourir à des indicateurs quantitatifs. Ceux-ci accélèrent considérablement la prise de décision, en

\footnotetext{
${ }^{3}$ Le discours du Président de la République au monde de la recherche, le 22 janvier 2009, fut, à cet égard, tout à fait symptomatique.

4 «L'évaluation par les pairs pose des problèmes pratiques liés à la lourdeur d'un examen approfondi des dossiers, amplifié par le nombre excessif des évaluations demandées de toute part. » [Académie des Sciences, 2011].

${ }^{5}$ Pour y avoir appartenu, les considérations parfois critiques sur le fonctionnement du CNU-5 ne mettent en cause ni le sérieux, ni la sincérité, ni le dévouement et le sens du service public de la très grande majorité de ses membres.

${ }^{6}$ Encore les 24 membres de rang A de la $5^{\text {ème }}$ section sont-ils, du fait de l'agrégation des universités, presque totalement exonérés de la qualification des Professeurs.

${ }^{7}$ Et ce d'autant plus que, lorsque les évaluateurs sont eux-mêmes chercheurs, le temps consacré à l'évaluation de leurs collègues est pris sur leurs propres activités de recherche.
} 
réduisant le temps nécessaire au traitement de chaque dossier, en "évacuant" la complexité des appréciations des rapporteurs, en simplifiant les hiérarchisations et classements, et surtout, en limitant, voire en supprimant, les débats et les controverses. Comme dans bien d'autres domaines, compter permet de ne pas débattre, et se révèle infiniment plus simple et plus rapide.

Il convient enfin de souligner le rôle des chercheurs eux-mêmes, dans le renforcement de l'évaluation quantitative de la recherche. D'abord, parce qu'en vertu du principe traditionnel d'évaluation par les pairs, ${ }^{8}$ ils sont souvent évaluateurs. Mais aussi, parce qu'ils ont globalement (du moins en économie), joué sincèrement le "jeu" qui leur était proposé. Si l'esprit de compétition, dont on ne voit guère pourquoi il serait moins développé chez les chercheurs que chez les autres individus, fut longtemps encadré par les normes et conventions traditionnelles du monde la recherche, la montée en puissance de la logique manageriale a conduit à le libérer et à l'encourager. Les chercheurs ont ainsi activement contribué au renforcement de la compétition symbolique et statutaire, ainsi qu'à l'émergence et à la montée en puissance d'une compétition économique et bibliométrique de plus en plus exacerbée.

L'esprit de compétition et la soif de classement des chercheurs ont ainsi convergé avec la logique manageriale et le poids des contraintes matérielles, pour pousser à la généralisation et à la systématisation de l'évaluation quantitative de la recherche. Au sein des sciences sociales, l'économie, par nature familière du quantitatif, et depuis longtemps travaillée par un fantasme de rapprochement avec les sciences "dures", s'est logiquement retrouvée à la pointe du mouvement.

Du point de vue du manager de la recherche, de l'évaluateur ou du chercheur, le recours systématique, voire exclusif, aux indicateurs quantitatifs et aux classements qui en découlent, présente un quadruple intérêt ${ }^{9}$ : simplifier et rationaliser le travail d'évaluation, circonscrire les débats et controverses, donner une apparence d'objectivité aux classements obtenus, et "cardinaliser" les objectifs de la compétition des chercheurs. C'est ainsi que, depuis une douzaine d'années, le CNU-5 s'est doté, pour la qualification des MCF, d'une "grille d'évaluation » comportant dix indicateurs, dont sept purement quantitatifs, les autres l'étant potentiellement. ${ }^{10}$ Ou que la liste recensant et classant (à l'origine, par un nombre d'étoiles) les revues d'économie et de gestion, établie par la section 37 du CNRS, a généré, dès sa première publication en juillet 2004, une véritable "course aux étoiles". Objectif désormais prioritaire de bien des chercheurs, qui le font fréquemment figurer sur leur curriculum vitæ, le "nombre d'étoiles CNRS" est, du point de vue de l'évaluateur ou du manager, un outil de classement particulièrement simple et pratique. Même si le CNU-5 terminant actuellement son mandat a repoussé de justesse l'utilisation explicite d'un cardinal de ce type pour les promotions des enseignants-chercheurs, ${ }^{11}$ la liste CNRS-37 n'en demeure pas moins une référence implicite, et elle a sert de base à l'établissement de "scores de publication" par le jury du concours d'agrégation, depuis 2008. Elle est aussi devenue la référence pour l'évaluation des économistes et de leurs laboratoires par l'AERES, et joue un

\footnotetext{
${ }^{8}$ Même si les récentes réformes en France (AERES, Comités de sélection...) s'affranchissent clairement de ce principe.

${ }_{9}^{9}$ Un parallèle vient immédiatement à l'esprit : celui des multiples sports de compétition dont les résultats sont (tout ou partie) liés à une notation par des «juges». Le recours à des «grilles » de notation de plus en plus précises permet de réduire, à la fois, la marge de manœuvre des juges, les possibilités de collusion, et les réclamations ou récriminations des compétiteurs.

${ }^{10}$ Par exemple, la mention de thèse ou la réalisation de stages post-doctoraux pourraient aisément être cardinalisées. Pour le détail de cette grille, voir [Beaumais, 2007, annexe 1].

${ }^{11}$ Session du 26 mars 2010.
} 
rôle déterminant dans l'attribution des financements, voire, pour dans un nombre croissant d'institutions, dans la rémunération individuelle des chercheurs (sous forme de primes).

Le fait que les concepteurs et initiateurs de cette liste aient été très largement débordés par son succès et par les usages qui en ont été faits [Pontille, Torny, 2010], illustre à la fois, la fascination qu'opèrent les indicateurs quantitatifs ${ }^{12}$ sur les chercheurs, évaluateurs et managers, et la puissance que ceux-ci confèrent, par la convergence de leurs comportements, à la dynamique du REQ.

\section{De l'évaluation par les pairs à l'évaluation "par les revues et les listes"}

Concrètement, l'affirmation du REQ s'est traduite par de profondes transformations des instruments, du processus, et du travail d'évaluation. Ces transformations se sont articulées avec un "déplacement" de la substance du pouvoir d'évaluation.

$\mathrm{Au}$ fur et à mesure que s'affirmait le REQ, les indicateurs ont fait l'objet de multiples perfectionnements et innovations, tel le h-index [Hirsch, 2005], devenu, en quelques années, l'alpha et l'oméga de nombre de chercheurs, en premier lieu aux États-Unis, et plus vite en sciences "dures" qu'en sciences humaines et sociales. Comme tout indicateur, le h-index souffre d'un certain nombre d'insuffisances, et a donc fait l'objet de multiples critiques. ${ }^{13}$ Sans entrer dans les détails, on peut cependant noter que le h-index, comme le g-index [Egghe 2006] censé en corriger certains biais, ${ }^{14}$ et comme tous les autres instruments de mesure de la productivité académique des chercheurs, repose, en dernier ressort, sur deux données : le nombre de publications (éventuellement pondéré par le rang), et le nombre de citations. Au fond, le débat technique sur les indicateurs est un débat sur la meilleure façon de construire et de combiner ces deux données. Or, il est essentiel de remarquer que le degré de perfectionnement de cette combinaison n'a d'impact direct, ni sur la fascination pour les indicateurs, ni sur la puissance du REQ; celui-ci peut fort bien prospérer sur la base d'indicateurs élémentaires. C'est ainsi que le CNU-5, se contente de dénombrer les publications (et communications) de chaque candidat, et de les classer en différentes catégories; ou que le "nombre d'étoiles CNRS" d'un chercheur n'est, arithmétiquement parlant, qu'une somme (parfois pondérée par certaines instances d'évaluation).

Il convient en outre de rappeler que ces indicateurs, aussi perfectionnés et raffinés soient-ils, reposent tous, in fine, sur des données dont le recueil et la construction sont, dans bien des cas, pour le moins approximatifs. C'est ainsi, que les deux grilles remplies (par chacun des deux rapporteurs) pour chaque candidat à la qualification des MCF par le CNU-5, diffèrent fréquemment, en particulier sur les indicateurs quantitatifs, alors même que ces indicateurs sont élémentaires.

"Il est important d'insister sur le fait que nombre d'analyses bibliométriques sont erronées en raison d'un mauvais recueil des données, d'erreurs dans le calcul des indicateurs, ou d'une interprétation inexacte. »[Académie des Sciences 2011]

C'est pourquoi on ne s'attardera pas sur la critique technique des indicateurs, certes nécessaire, mais qui, bien souvent, tend à éclipser la réflexion sur la logique et le

\footnotetext{
${ }^{12}$ On peut noter que le même phénomène a touché bien d'autres indicateurs ou classements (h-index, classement de Shanghai ....).

${ }^{13}$ Voir, par exemple : [Gingras, 2008], ou, plus ironiquement, le chercheur fictif Ike Antkare, créé par Cyril Labbé (Grenoble 1), et ayant atteint un h-index de 94 (avril 2010), soit le $21^{\text {ème }}$ rang mondial, toutes disciplines confondues, Einstein n'atteignant, lui, que le $36^{\text {ème }} \mathrm{rang}$ ! ( http://rr.liglab.fr/research_report/RR-LIG-008.pdf )

${ }^{14}$ En particulier en sur-pondérant les articles très cités, et très longtemps après leur publication.
} 
fonctionnement concret du REQ ; la critique des indicateurs, aussi utile soit-elle, ne saurait se substituer à celle de l'évaluation par indicateurs.

La montée en puissance du REQ s'est traduite par une profonde évolution du travail concret des instances d'évaluation. Le cadre traditionnel de collectifs de scientifiques émettant, après lecture et discussion, des avis (par nature discutables) sur les travaux qui leur sont soumis, se réduit de plus en plus aux apparences. Désormais, dans la plupart des cas, leur tâche effective se limite à opérer la synthèse d'un certain nombre d'indicateurs.

C'est ainsi que, concernant les promotions des MCF et des Professeurs (PR), le CNU5 statue sur la seule base des dossiers et indicateurs, sans disposer des travaux (ou même d'une sélection de travaux) des candidats. Il en va de même pour les promotions dite "locales", au sein de chaque université. Ça n'est que pour la qualification des MCF, qu'il est demandé aux candidats de fournir une sélection de leurs travaux. Chaque rapporteur dispose alors d'environ six semaines (dont les fêtes de fin d'années) pour, en plus de ses activités habituelles d'enseignant-chercheur, "traiter" une quinzaine de dossiers, c'est-à-dire, établir les indicateurs au vu des données figurant dans les dossiers, puis lire et apprécier les travaux (thèse + articles) de chaque candidat, et enfin rédiger un rapport sur chacun d'entre eux. Cependant, les rapporteurs savent tous que leur appréciation sur les travaux d'un candidat n'a qu'un impact marginal sur la décision du CNU-5. Une étude [Beaumais 2007] réalisée sur les qualifications de 2005 et 2006 1'a clairement montré. Pour obtenir la qualification aux fonctions de MCF, deux critères sont largement prépondérants : la mention de thèse "Très Honorable avec les Félicitations du jury"; au moins une publication dans une revue référencée par la base Econlit (American Economic Association).

Concernant les recrutements à l'échelle locale, les pratiques concrètes varient évidemment selon les universités et les disciplines. Mais les conditions matérielles (délais, nombre de candidats, nombre et compétence disciplinaire des membres des Comités de sélection...) poussent souvent, au moins pour certaines étapes, ${ }^{15}$ à procéder sans lecture des travaux. Les "bonnes intentions", telles celles exprimées par l'Académie des Sciences, ${ }^{16}$ ne pèsent guère face à la réalité matérielle.

L'AERES et l'ANR, par leur composition et leurs critères d'évaluation, constituent, à ce jour, l'aboutissement de cette logique : une évaluation sans lecture ni appréciation des travaux. Dès lors, le travail d'évaluation (la synthèse d'indicateurs) ne nécessitant aucune compétence sur le fond, le confier à des chercheurs de la discipline constituerait un évident gaspillage de ressources. D'ailleurs, quel membre du CNU-5, noyé dans le dossier d'un candidat, et cherchant désespérément à en extraire les données nécessaires à l'établissement de la grille d'évaluation, ne s'est jamais dit que ce travail ardu ne nécessite pas la moindre compétence en économie?

Les différentes instances officielles d'évaluation des chercheurs et de la recherche tendent ainsi à n'être que de simples «chambres » de synthèse d'indicateurs. De ce fait, et malgré les apparences institutionnelles, la substance du pouvoir d'évaluation leur échappe. Ainsi, dans le cas de la qualification aux fonctions de MCF en économie, le rôle prépondérant appartient, in fine, aux jurys de thèse et aux comités éditoriaux des revues référencées par la base Econlit.

Plus généralement, l'évaluation par le nombre d'articles conduit inéluctablement à transmettre le pouvoir d'évaluation aux boards et referees des revues; la prise en compte du

\footnotetext{
${ }^{15}$ La sélection des candidats à auditionner se fait fréquemment sans accès aux travaux.

${ }^{16}$ "Les indicateurs bibliométriques n'ont pas de valeur au début de la carrière scientifique et ne doivent donc pas être utilisés pour les recrutements, sauf lorsqu'il s'agit du recrutement de seniors. (...) La bibliométrie doit exclusivement être utilisée en association avec une évaluation qualitative. »
} 
rang des publications en redistribue une partie aux différentes institutions, privées ou publiques, recensant et classant les revues. En simplifiant à l'extrême, pour un économiste français, la substance du pouvoir d'évaluation est désormais entre les mains des comités éditoriaux des revues, à hauteur du classement de la revue dans la liste établie par la section 37 du CNRS. Du score qu'il atteindra dans la "course aux étoiles", dépendront sa carrière (à travers le CNU, le CNRS, l'AERES...), et sa capacité à obtenir des financements (universités autonomes, ANR, fonds européens...).

Finalement, le REQ, qui doit sa puissance à la conjonction des comportements de tous les acteurs concernés, vise à maximiser la productivité académique des chercheurs, par la systématisation de la compétition, ${ }^{17}$ et l'établissement de classements explicites ou implicites reposant sur des indicateurs quantitatifs. Dans le cadre d'un tel régime, la substance du pouvoir d'évaluation revient, in fine, aux revues et aux listes.

\section{Incitations, biais, contraintes}

Du point de vue des chercheurs, le REQ agit comme un système d'incitations visant à les encourager à participer à la compétition, en particulier bibliométrique et économique. Certes, parmi les chercheurs, comme au sein de tout groupe d'individus, il en existe de plus ou moins perméables, ou de plus ou moins rétifs, aux incitations. Mais globalement, au fur et à mesure de la montée en puissance du REQ, les chercheurs ont naturellement été amenés à faire évoluer leurs comportements (objectifs, travail concret...), et donc le produit de leur travail. C'est pourquoi on tentera de repérer les particularités (biais, contraintes) comportementales générées par le REQ, et de montrer comment elles influent sur la nature et le contenu des recherches menées. On s'attachera, en particulier, à montrer comment le REQ pèse sur les capacités d'innovation et de renouvellement des disciplines, et spécifiquement de l'économie.

Idéalement, le «programme » du chercheur se limite à un objectif unique : consacrer toutes les ressources dont il dispose, à faire progresser sa discipline. Bien entendu, la réalité n'a jamais été aussi simple; l'intérêt individuel des chercheurs et les relations concurrentielles qu'ils entretiennent n'ont pas attendu l'époque contemporaine pour interférer avec cet idéal. Cependant, avec le REQ, il ne s'agit plus d'interférences, mais de substitution ; désormais, les progrès de la discipline ne font plus partie du programme individuel du chercheur. ${ }^{18}$ Qu'il s'agisse de sa carrière, de sa reconnaissance académique, de sa capacité à obtenir des contrats de recherche, de la prospérité et du devenir de son laboratoire, et même de son université, ${ }^{19}$ l'objectif exclusif de l'individu-chercheur rationnel est désormais de maximiser «ses » indicateurs. Il serait même possible de ramener le programme du chercheur à la maximisation d'un indicateur synthétique unique, tel que le h-index ou le nombre

\footnotetext{
${ }^{17}$ Pour persévérer dans la métaphore sportive (cf : note 9), en l'occurrence celle des transferts de joueurs ou d'entraîneurs dans le football professionnel, on peut évoquer le cas de l'Université Paris-7, qui, après s'être doté d'un Président, star médiatique de la génétique et "recruté à l'extérieur" du corps professoral de l'université, a récemment lancé une campagne de communication pour se vanter d'avoir recruté un prix Nobel américain (sans mentionner toutefois le prix du transfert!).

${ }^{18}$ De la même façon que la quantité produite est absolument indifférente au producteur rationnel, exclusivement préoccupé de maximiser son profit.

${ }^{19}$ Une partie du budget des universités étant désormais liée au nombre de chercheurs «publiants », au sens des critères de l'AERES.
} 
d'étoiles CNRS. Dans la suite du propos, on considérera cependant que le chercheur vise à maximiser le nombre et le rang de ses publications, et, à un moindre degré, le nombre de citations dont ses publications font l'objet.

\section{2-1) Biais comportementaux}

Dans ce cadre, le travail concret des chercheurs est affecté par de nombreux biais comportementaux, dont l'impact sur le produit du travail est certes variable, mais dont la combinaison pèse lourdement sur les contenus, les méthodes et les résultats des recherches entreprises, et donc sur le devenir des différentes disciplines. Ces biais ne sont évidemment pas tous nouveaux ; lorsque ce n'est pas le cas, le REQ leur insuffle une puissance qui, elle, est nouvelle.

On peut, dans un premier temps, mentionner quelques biais qui, à l'instar de ceux affectant les indicateurs, remettent en cause la pertinence et la rigueur des résultats de l'évaluation, mais n'ont, à première vue, pas d'impact direct sur le contenu des travaux. Ainsi, l'objectif de maximisation du nombre de publications conduit-il à la multiplication des signatures collectives, ${ }^{20}$ dont il n'est pas toujours avéré qu'elles correspondent à une absolue nécessité scientifique, et à une répartition du travail parfaitement équilibrée entre les signataires, sachant qu'en économie, l'ordre des auteurs (alphabétique) ne dit rien de leurs contributions respectives. Quant à l'objectif de maximisation du nombre de citations, c'est tout naturellement qu'il conduit à la multiplication des auto-citations ${ }^{21}$ et des effets de réseaux (citations réciproques par collusion); et c'est tout aussi naturellement que les doctorants, déjà parfaitement conscients des impératifs du métier auquel ils se destinent, n'oublient jamais, dans leurs premières publications, de citer abondamment leur directeur de recherche. De façon plus anecdotique, tout chercheur sait qu'il est habile et "de bonne guerre", dans un article soumis à une revue, de citer, aussi fréquemment que possible, les membres du board et les articles ${ }^{22}$ de cette revue. Enfin, même si l'évolution technologique a sans aucun doute joué un rôle, la pression à la publication induite par le REQ a favorisé la banalisation du plagiat, régulièrement dénoncée depuis quelques années. ${ }^{23}$

Ces quelques exemples, sans impact apparent sur le produit du travail de recherche, donnent cependant un premier aperçu de l'ampleur de la tâche que constitue, pour le chercheur, la mise en œuvre d'une stratégie efficace dans le cadre du REQ. Et dans cette perspective, d'autres biais affectent directement le travail de recherche en tant que tel, et donc, la nature et la qualité du produit.

Tout d'abord, pour maximiser la probabilité de publication d'un article et, à plus long terme, son nombre de publications, le chercheur s'efforcera, naturellement, de rendre les résultats qu'il a obtenus aussi attractifs que possible. Or, il n'existe pas de frontières précises, mais un continuum, entre la mise en valeur habile des résultats et la falsification (terme qu'on réserve ici aux cas extrêmes). Si la falsification pure et simple, heureusement exceptionnelle, existait bien avant le REQ, celui-ci favorise inévitablement les pratiques "intermédiaires" consistant à "s'arranger" avec certaines données, à en ignorer d'autres, à embellir certains résultats et à en passer d'autres sous silence. Pour s'en tenir à l'économie, qui n'a jamais été

\footnotetext{
${ }^{20}$ De ce point de vue, les sciences "dures" sont en avance sur les sciences sociales.

${ }^{21}$ C'est bien pourquoi certains indicateurs « isolent » et excluent les auto-citations.

${ }^{22}$ Ce qui contribuera, en cas de publication, à accroître l'Impact Factor de ladite revue.

${ }^{23}$ Voir, par exemple, les sites : http://www.plagiat-recherche.fr ; http://responsable.unige.ch ; http://archeologiedu-copier-coller.blogspot.com ...
} 
tenté, ne serait-ce qu'un instant, de recourir à de "petits arrangements" permettant, bien sûr à la marge, de rendre plus "présentables" les résultats d'un test économétrique ? De même, avec la vogue actuelle de l'économie expérimentale, qui n'a jamais éprouvé le moindre doute sur le sérieux, la rigueur, les conditions concrètes et la fiabilité des résultats de certaines expériences, pourtant publiées?

Les chercheurs ne sont pas moins tentés que les autres individus par des pratiques "biaisées" leur permettant d'atteindre plus aisément leurs objectifs. ${ }^{24}$ Le REQ, qui renforce très sensiblement ces tentations, est donc générateur d'incertitude quant à la qualité et à la fiabilité du produit du travail des chercheurs.

Second biais particulièrement lourd de conséquences, l'ensemble des pratiques qu'on peut regrouper sous le terme de "sur-publication ». Celle-ci découle bien sûr de la logique générale du REQ, mais aussi du fait qu'à ce jour, dans le programme du chercheur, le nombre (et le rang) de publications apparaît clairement prioritaire par rapport au nombre de citations. Pour un économiste français, le nombre de citations n'intervient ni dans les indicateurs retenus par le CNU-5, ni dans ceux retenus par l'AERES. Plus généralement, même lorsqu'il en est tenu compte, le nombre de citations est généralement un indicateur de second rang. Il en est ainsi dans le calcul du h-index, qui se veut pourtant être un indicateur synthétique : au cours d'une période donnée, un chercheur ayant publié six articles, ayant fait l'objet de six citations chacun, a un h-index de 6 ; un chercheur ayant publié deux articles, ayant fait chacun l'objet de centaines de citations, a un h-index de 2 , et se révèle donc trois fois moins $\mathrm{h}$ performant que le premier. Enfin, le nombre de publications est un des facteurs dont dépend le nombre de citations. Bref, tout pousse le chercheur à se focaliser sur le nombre de ses publications. C'est de cette obsession que résultent les pratiques de sur-publication.

Il est ainsi logique de tronçonner les résultats d'une recherche en autant d'éléments publiables que possible, quitte à en affaiblir la cohérence d'ensemble; c'est ainsi que de plus en plus de thèses en économie sont, implicitement ou explicitement, des recueils d'articles. De même, il apparaît rationnel, d'établir successivement, plusieurs "versions" ou "évolutions" d'un contenu similaire, ne différant que par des adaptations et modifications aussi mineures ou formelles que possible, puis, au fur et à mesure de leur rédaction, de les soumettre à des revues différentes. Plus généralement, le REQ pousse le chercheur rationnel à consacrer toute son énergie à la production accélérée de "nouveautés éditoriales », aussi marginales et insignifiantes soient-elles du point de vue des progrès de sa discipline. [Cordonnier 2011]

D'autre part, la course à la publication pousse au renforcement de la spécialisation et de son inertie; le chercheur est, de fait, incité à se spécialiser dans un créneau précis, aussi porteur que possible, puis à exploiter le filon tant qu'il offre des opportunités de publication, puisque, sauf cas particulier, ${ }^{25}$ un changement de spécialisation se traduirait par une réduction, au moins temporaire, de son rythme de publication (coûts de transfert).

Apprécier l'ampleur de la sur-publication générée par le REQ est évidemment très difficile. Un rapport [Bosquet, Combes, Linnemer. 2010], commandé par le Ministère de l'Enseignement Supérieur et de la Recherche, en fournit un ordre de grandeur, pour l'économie. Il constate que, depuis le milieu des années 1990, le nombre annuel d'articles "Econlit" publiés par des chercheurs français a approximativement triplé, mais que la "qualité moyenne" ${ }^{26}$ des publications n'a, elle, pas progressé. Parallèlement, les auteurs soulignent que l'accroissement du rythme de publication est essentiellement le fait des "entrants", c'est à

\footnotetext{
${ }^{24}$ Dans la mesure où ces « arrangements », sont parfois à l'origine de polémiques, ils peuvent même contribuer à accrôitre le nombre de citations de l'auteur.

25 "Ancien" filon passé de mode, "nouveau" filon émergent et porteur.

${ }^{26}$ Calculée sur la base d'un classement des revues en fonction du nombre de citations dont leurs articles font l'objet.
} 
dire, des générations de chercheurs ayant été, dès le début de leur carrière, confrontées à la montée en puissance du REQ. On retrouve logiquement cette distorsion générationnelle dans les résultats des évaluations fondées sur des indicateurs bibliométriques ; lors de la session du CNU-5 de mai 2009, parmi les huit promus au premier échelon de la classe exceptionnelle des Professeurs, sept avaient moins de cinquante ans. ${ }^{27}$

Du point de vue des progrès de la connaissance scientifique, la sur-publication, et les innombrables redondances qu'elle entraîne, sont l'un des principaux gaspillages de ressources générés par le REQ : gaspillage lié à la spécialisation et à son inertie ; mais aussi, pour les lecteurs, de plus en plus submergés par une marée de publications, dont l'apport unitaire est souvent très réduit ; et surtout pour les auteurs, par le temps et l'énergie consacrés à multiplier les articles et les applications, plutôt qu'à essayer de progresser sur le cœur de leur démarche. Plus généralement, la pression induite par le REQ conduit le chercheur rationnel à consacrer un temps considérable à communiquer sur son travail, que ce soit par des participations à des colloques, indispensables pour accroître ses probabilités de publication et de citation, ou par la rédaction d'articles aussi nombreux que possible. Au fond, le chercheur rationnel consacre infiniment plus de temps et d'énergie à faire en sorte de publier, qu'à chercher. Il convient de souligner les effets de ce biais sur les jeunes chercheurs, auxquels les impératifs de publication interdisent désormais de prendre le temps nécessaire pour se constituer une culture générale de leur discipline, pour construire peu à peu leur propre démarche, leur propre cadre de pensée.

Si elle accroît la productivité académique telle qu'elle est aujourd'hui mesurée, la surpublication pèse ainsi négativement sur la productivité scientifique ; en outre, en favorisant l'inertie des spécialisations, elle réduit les capacités de renouvellement, et contribue ainsi à asphyxier ou stériliser la dynamique de la connaissance scientifique. Il y a quelques années, l'éminent géomètre franco-russe Mikhaïl Gromov (prix Abel 2009) décrivait ainsi les effets dévastateurs de la sur-publication et de la pression induite par le REQ, pour l'avenir des mathématiques :

« En Amérique, beaucoup de mathématiciens deviennent rapidement limités car ils sont tenaillés par l'urgence de publier et d'obtenir des subventions. S'ils ne sont pas experts dans un domaine, ils n'en reçoivent pas. Cela les rend très étroits d'esprit, tant dans leur savoir que dans leur attitude à l'encontre d'autres savoirs (...). En France, les choses vont beaucoup mieux en raison de l'éducation héritée de Bourbaki, qui a développé un enseignement inter-mathématique. Un mathématicien français est beaucoup plus instruit en mathématiques qu'un mathématicien américain. » In : [Ripka, 2002].

\footnotetext{
${ }^{27}$ «L"'excellence scientifique" comme prétexte au scientisme», lettre ouverte par des membres élus de la section Économie du CNU, 21 janvier 2010.
} 


\section{2-2) Contraintes formelles}

Aux biais affectant le comportement du chercheur s'ajoutent des contraintes formelles encadrant la présentation/publication des résultats de son activité, et qui conditionnent, ellesaussi, le contenu voire la nature des travaux entrepris.

Alors que l'ouvrage avait longtemps été la forme privilégiée de diffusion des résultats de la recherche en sciences sociales, la montée en puissance du REQ a conduit, en particulier en économie, à s'aligner sur les pratiques des sciences "dures", en faisant de l'article (dans une revue à comité de lecture) la forme quasi-exclusive de la production académique. C'est ainsi que dans le processus de qualification des MCF par le CNU-5, l'impact de la publication d'un ouvrage (même sous signature individuelle) est tout à fait négligeable, alors que celui d'un article dans une revue référencée Econlit se révèle décisif [Beaumais, 2007].

En sciences sociales, la contrainte concrète la plus immédiate que fait peser le format article, est la limitation stricte de la «taille» des textes produits. Si chaque revue est libre de définir la limite qu'elle souhaite, il existe de fait une norme courante qui, en économie, est de l'ordre de 50 à 90000 signes. Quels que soient ses raisons et ses éventuels effets positifs, cette contrainte conditionne largement le travail des chercheurs.

Tout d'abord, le format article confronte le chercheur à un dilemme : s'il souhaite rendre compte précisément et rigoureusement des différentes étapes de son raisonnement, il est condamné à se limiter à un objet étroitement délimité, à un champ réduit, voire ponctuel ; s'il souhaite aborder un champ élargi, il est condamné à un propos très synthétique, tendant à gommer les nuances, les détails et les précautions qui caractérisent la démarche scientifique. En économie, où la première option a clairement été privilégiée par les revues et les chercheurs, la prépondérance du format article a ainsi joué un rôle important dans le renforcement de la spécialisation des chercheurs et du morcellement de la discipline.

Parallèlement, le format article, en incitant les économistes à privilégier les outils et méthodes qui s'y adaptent le plus aisément, stimule certains domaines de la pensée économique, en déstabilise d'autres, et, par conséquent, influe directement sur la dynamique et la structuration de la discipline. Il a ainsi contribué au recours de plus en plus systématique à la formalisation mathématique et aux tests économétriques, et, plus récemment, au succès de l'économie expérimentale. À l'inverse, alors que d'éminents économistes, tel Maurice Allais, [Allais, 2009] dénoncent une "spécialisation outrancière », plaident pour un effort général de synthèse, et regrettent le manque de culture historique des jeunes économistes, ${ }^{28}$ le format article favorise une histoire de la pensée économique de plus en plus spécialisée, voire "ponctuelle", et philologique; quant à l'économie historique, la quasi-impossibilité de présenter un travail de synthèse dans le format article entrave considérablement son intérêt pour les non-spécialistes, et donc son rayonnement.

On peut enfin remarquer que le format article est porteur d'une vision très déformée, voire artificielle, du travail scientifique. Les interrogations qui demeurent, les doutes, les fausses pistes et les errances de la démarche scientifique sont, en effet, de plus en plus systématiquement passés sous silence. D'une part, les revues ne publient pas les fausses pistes et les errances, d'autre part, le format article ne laisse guère l'opportunité d'en parler. Sans compter que la plupart des chercheurs sont convaincus, à tort ou à raison, qu'évoquer les méandres de la démarche scientifique réduit la probabilité de publication.

\footnotetext{
${ }^{28}$ Voir aussi, plus récemment [Shiller 2011] «Specialization coupled with strong pressures within academia leads to a situation in which adademics often feel that they just do not have time to ponder broad issues and learn even basic simple facts outside their specialty. Their general knowledge may be embarrassingly limited (...).»
} 
Les chercheurs français sont confrontés à une autre contrainte formelle lourde ; celle de la langue, et plus précisément de l'anglais. Certes les effets de cette contrainte linguistique paraissent, a priori, limités pour les sciences "dures"; la présentation d'une démonstration mathématique ne dépend que très marginalement de la langue utilisée, et n'exige pas de son auteur une parfaite maîtrise de cette langue. Cependant, en sciences humaines et sociales, où la langue écrite demeure le principal véhicule de la pensée, rendre compte rigoureusement de phénomènes complexes exige une maîtrise très précise de la langue utilisée, et le passage d'une langue à l'autre soulève fréquemment des problèmes de fond, qui donnent lieu à d'innombrables débats de traduction. ${ }^{29}$

En économie, la contrainte linguistique est particulièrement forte ; l'immense majorité des revues référencées sont anglophones. Surtout, la prépondérance des revues anglophones est d'autant plus forte qu'on s'élève dans la hiérarchie des revues, telle qu'elle est définie par les listes et les classements. Ainsi les six revues économiques ${ }^{30}$ classées $1^{*}$ (rang le plus "élevé") dans la liste CNRS-37 sont anglophones. Quant aux 58 (sur 705) revues francophones classées, 40 d'entre elles sont en catégorie 4, la plus faible [Audier, 2009]. On peut aussi remarquer que la seule revue francophone classée en catégorie 1 (Annales, Histoire, Sciences sociales) ne publie, paradoxalement, que très peu d'articles d'économie.

Comme le format article, la contrainte linguistique ne pèse pas de la même façon sur les différents domaines de l'économie, ${ }^{31}$ et influe donc sur la dynamique et la structuration de la discipline.

\section{Normalisation, conformisme, standardisation}

Ces biais et contraintes encadrant étroitement le travail concret des chercheurs, s'inscrivent dans un processus général de normalisation des comportements induit par le REQ. Dans ce processus, les revues, auxquelles a, de fait, été délégué le pouvoir d'évaluation, sont le «lieu » de production des normes et des modes, que le conformisme et les incitations du régime d'évaluation poussent les chercheurs à incorporer. Il en résulte une tendance à la standardisation de la production scientifique, qui affaiblit les capacités d'innovation et de renouvellement des disciplines, et, plus largement, la dynamique de la connaissance scientifique.

En économie, ce processus prend des formes originales. Par rapport aux autres sciences sociales, la normalisation instrumentale y joue un tel rôle, qu'on peut parler de dérive instrumentaliste; insensiblement, dans la démarche scientifique de bon nombre d'économistes, "l'outil est devenu l'objet". On peut citer l'un des représentants emblématiques de cette dérive, Steven Levitt : "L'économie [est] un jeu d'outils avant d'être un sujet en soi $\gg .32$

Dans un milieu professionnel par nature sujet au conformisme et aux jeux de réseaux, ne serait-ce que par le principe de cooptation, le pouvoir des revues dans le REQ se déploie à travers le réseau de chercheurs qui gravite autour de chaque revue. Le centre de gravité de ce

\footnotetext{
${ }^{29}$ Qu'on songe aux interminables querelles sur la traduction des œuvres d'auteurs tels que Karl Marx ou Sigmund Freud.

${ }^{30}$ Les deux autres revues classées $1 *$, également anglophones, sont des revues de gestion.

${ }^{31}$ Par exemple, l'économie mathématique et quantitative, comme les sciences "dures", n'est que peu "perturbée" par la contrainte linguistique.

${ }^{32}$ Interview accordé à Samuel Blumenfeld. Le Monde 2, 25 février 2006.

On peut également souligner la puissance de cette dérive instrumentaliste dans l'enseignement de l'économie.
} 
réseau est constitué par les membres du board et les referees. Bien sûr, en tant que chercheur, chacun d'entre eux, par son appartenance à d'autres réseaux professionnels, dispose, de fait, d'un réseau de relations, plus ou moins proches, qui lui est propre. C'est l'ensemble de ces relations avec le centre de gravité qui constitue le réseau d'une revue.

Or, appartenir au réseau gravitant autour d'une revue, directement ou par le réseau personnel d'un membre du board ou d'un referee, constitue, pour un chercheur, un avantage concurrentiel significatif pour publier dans cette revue, ne serait-ce que par l'accès privilégié à l'information sur les normes (explicites et implicites) de publication propres à la revue, ${ }^{33}$ ou aux éventuels colloques au sein desquels il est possible de faire connaître et de "roder" son article auprès des membres du réseau. Cela peut aussi permettre plus aisément de faire relire son texte, avant soumission, par un membre du réseau aussi éminent que possible, lequel sera bien sûr respectueusement remercié en début d'article, l'affichage d'un nom prestigieux ne pouvant nuire à la probabilité de publication.

Bien entendu, l'appartenance d'un chercheur au "centre de gravité" d'une revue n'est en rien exclusive. Être membre du board ou du corps des referees de plusieurs revues constitue, au contraire, un signe d'excellence pour le chercheur concerné, ainsi qu'un facteur de nature à faciliter ses publications et sa carrière. Il existe, de ce fait, un enchevêtrement inextricable des réseaux gravitant chacun autour d'une revue. Cependant, toutes les revues, et donc tous les réseaux, ne se valent pas ; les classements de revues, eux-même influencés par les processus d'auto-validation propres aux réseaux [Hodgson, Rothman, 1999], définissent une hiérarchie des réseaux calquée sur celle des revues. Une étude américaine [McKinney, Rupp 2002] en fournit une illustration presque caricaturale : afin «d'informer les étudiants et les administrateurs universitaires », les auteurs proposent un classement des sept meilleurs Départements d'économie aux Etats-Unis, sur la base des publications de leurs membres dans les cinq meilleures revues, le classement des revues étant établi à partir... du nombre d'articles signés par des auteurs appartenant aux sept meilleurs Départements !

$\mathrm{Au}$ sein de chaque discipline se constitue ainsi un enchevêtrement hiérarchisé de réseaux de revues, qui constitue le principal vecteur de diffusion, d'incorporation et de reproduction, des normes et des modes. C'est par son intermédiaire que s'opèrent la normalisation et la standardisation du travail des chercheurs.

En déléguant la substance du pouvoir d'évaluation aux revues, le REQ leur confie, du même coup, le pouvoir de définir les normes et les modes propres à chaque discipline. Chaque revue, par son positionnement (théorique, méthodologique...), par son domaine de référence, et par les membres de son réseau, est porteuse d'une certaine vision de la discipline, de ses paradigmes, de ses thématiques, de ses méthodes. À une échelle plus globale, c'est à travers l'enchevêtrement hiérarchisé de réseaux, au sein duquel les revues les mieux classées sont logiquement les plus influentes, qu'est générée la vision dominante et normalisatrice de la discipline, vision bien sûr évolutive. Ce sont ainsi les revues, et en particulier les mieux classées, qui orientent et canalisent les évolutions et le devenir de chaque discipline. En économie, où la suprématie des revues anglo-saxonnes, et en particulier américaines, frise le monopole, ${ }^{34}$ le REQ a considérablement renforcé l'emprise, déjà forte, des normes et de la vision "anglo-saxonnes" de la discipline [Hodgson, Rothman, 1999]. Les économistes contemporains sont ainsi, beaucoup plus que les générations précédentes, touchés par une normalisation de la discipline à l'échelle mondiale.

\footnotetext{
${ }^{33}$ De la même façon que, pour un doctorant se destinant à la carrière universitaire, le contact direct avec un ou des membres du CNU, lui permet d'optimiser son dossier de candidature à la qualification (ne serait-ce qu'en le présentant de façon à faciliter le travail des rapporteurs).

${ }^{34}$ Des six revues classées $1 *$ par le CNRS-37, cinq sont américaines, et une anglo-américaine.
} 
Ce processus de normalisation est particulièrement puissant, car, loin de tenter d'y résister, le chercheur rationnel, attentif aux incitations induites par le REQ, consacrera, au contraire, d'importantes ressources à l'incorporation des normes produites par les revues. Pour accroître sa probabilité de publication dans une revue, il s'efforcera naturellement d'appréhender et de faire siennes la vision de la discipline et les normes thématiques, méthodologiques, instrumentales et rédactionnelles, propres à ladite revue. Et pour multiplier le nombre et améliorer le rang de ses publications, il sera logiquement conduit à tout faire, pour coller au plus près de la vision dominante de la discipline et des ses évolutions.

Certes, rien n'interdit à des chercheurs ne se reconnaissant pas dans la vision dominante, d'organiser séminaires et colloques, et de publier des ouvrages, individuels ou collectifs ; cependant, au moins en économie, ces formes de production scientifique sont de plus en plus ignorées par les instances d'évaluation. ${ }^{35}$ De même, rien n'interdit d'éditer des revues porteuses d'une vision hétérodoxe de la discipline, mais leur référencement et leur classement dans les listes nuiront inévitablement à la carrière des auteurs y publiant leurs travaux. Le REQ tend ainsi à exclure les chercheurs prétendant échapper à la vision dominante, en les condamnant à une carrière de second rang. [Postel 2011]

Le REQ, conjugué à la systématisation des financements sur projets, rend ainsi le conformisme rationnel pour le chercheur. Logiquement, celui-ci ne produira que ce qui a une forte probabilité de publication, et ne se lancera que dans des projets dont les résultats sont prévisibles, programmables, ${ }^{36}$ rapides et publiables. Il en résulte un comportement général d'autolimitation, visant à se conformer aux normes et impératifs des revues, et générant une tendance lourde à la standardisation des ambitions, des perspectives, des paradigmes, des thématiques, des méthodes et outils, des pratiques rédactionnelles...

Bien entendu, d'une discipline à l'autre, la pondération des différents "axes" de la standardisation est variable. Ainsi l'économie contemporaine est-elle très ouverte en matière de thématiques; de Gary Becker (Nobel 1992) à Steven Levitt (John Bates Clark Medal 2003), des économistes reconnus se sont penchés sur des questions tellement variées et apparemment peu économiques, qu'on parle aujourd'hui d' «économie saugrenue » [Levitt, Dubner 2005]. De même, à l'heure du défi posé par les répercussions de la crise financière et de la contrainte écologique, deux des six revues économiques les plus prestigieuses viennent de publier des articles sur des sujets tels que, les liens existant entre la violence conjugale et les résultats des matchs de football américain, ${ }^{37}$ ou l'avantage dont disposerait l'équipe tirant en premier, lors des séances de tirs au but qui concluent les matchs de football, en cas d'égalité après prolongations. ${ }^{38}$ À l'inverse, l'économie contemporaine souffre d'une très forte standardisation paradigmatique [Raveaud 2011]: la représentation walrassienne de l'économie comme ensemble de marchés interdépendants tendant vers l'équilibre. Elle est en outre, du fait de sa dérive instrumentaliste, particulièrement soumise aux modes instrumentales; à la vogue de l'économie quantitative de la fin du $20^{\text {ème }}$ siècle, s'est ainsi ajoutée, au début du $21^{\text {ème }}$, celle de l'économie expérimentale, ${ }^{39}$ voire de la neuro-économie. ${ }^{40}$

Cette standardisation des comportements et du travail des chercheurs est évidemment nuisible à leur créativité, à leur imagination, à leurs ambitions, à leur esprit critique, à leur

\footnotetext{
${ }^{35}$ Voir, par exemple : «L"“excellence scientifique” comme prétexte au scientisme », lettre ouverte par des membres élus de la section Économie du CNU, 21 janvier 2010.

${ }^{36}$ On ne peut s'empêcher de souligner à quel point, l'idée d'une démarche scientifique prévisible et programmable, relève de l'oxymore.

${ }^{37}$ [Card, Dahl. 2011] in : The Quarterly Journal of Economics.

${ }^{38}$ [Apesteguia, Palacios-Huerta. 2010] in : American Economic Review. On peut noter que les résultats de cette étude ont été contestés... par de nouvelles publications !

${ }^{39}$ L'évolution des thèses est, à cet égard, tout à fait symptomatique.

${ }^{40}$ Laquelle ne se définit, au fond, que par son outil : l'imagerie par résonance magnétique.
} 
goût du risque... En incitant les chercheurs à consacrer leur énergie et leur intelligence à l'incorporation des normes et à la satisfaction des critères d'évaluation, elle génère d'importants gaspillages de ressources, du point de vue des progrès de la connaissance scientifique. Le développement de la connaissance scientifique, les capacités d'innovation des chercheurs et le renouvellement des disciplines, sont ainsi étroitement canalisés et bornés.

\section{Appréciation vs évaluation}

Le REQ, par les biais comportementaux qu'il génère, par le pouvoir normalisateur qu'il délègue aux revues et aux listes, et en incitant le chercheur rationnel au conformisme, débouche sur une standardisation du travail des chercheurs, et donc du produit de leur travail. Visant à améliorer la productivité académique, il tend concrètement à asphyxier la dynamique de la connaissance scientifique. En paraphrasant Nietzsche, on pourrait dire qu'à trop vouloir accrô̂tre la productivité académique, le REQ affaiblit la productivité scientifique.

De façon qui n'est paradoxale qu'en apparence, le REQ se révèle ainsi être un obstacle important au renouvellement de la pensée économique, dont la crise financière récente et les préoccupations écologiques ont cruellement mis en évidence la nécessité. Promouvoir un tel renouvellement exige donc, parmi bien d'autres choses, d'enrayer la dérive quantitativiste qui affecte aujourd'hui le régime d'évaluation.

En ce qui concerne les chercheurs, dissiper l'illusion quantitativiste ne devrait pas poser de problèmes insurmontables ; ils savent tous que les progrès scientifiques ne sont pas affaire de nombre de publications, ni même de citations ; c'est ainsi que les deux lauréats 2010 de la Médaille Fields ont des "scores de citations" très différents, ${ }^{41}$ " alors qu'aucun mathématicien au monde ne se risquerait à y voir une disparité de niveau ». [Académie des Sciences 2011]. Les chercheurs savent tous que, bien souvent, les progrès les plus décisifs sont le fruit de «recherches orphelines », que le régime d'évaluation quantitative, par nature, ignore. Ils savent tous qu'en 1905, deux articles, sur l'effet photo-électrique et la relativité restreinte, ont suffi à Einstein pour révolutionner l'histoire de la physique. De même, tous les économistes savent que Ronald Coase (Nobel 1991) a bouleversé la pensée économique de la seconde moitié du $20^{\text {ème }}$ siècle, par deux articles publiés à 23 ans d'intervalle et longtemps ignorés, ou qu'il a suffi d'un article d'une page à John Nash (Nobel 1994), pour faire franchir à la théorie des jeux une étape décisive...

Ces constats n'empêchent pourtant pas la plupart des économistes de se satisfaire du REQ, ou de considérer comme perdue d'avance toute tentative de remise en cause. Quant aux rares résistances qui se font jour, elles tendent malheureusement à se cantonner à la défense des modalités "traditionnelles" d'évaluation, lesquelles n'étaient, faut-il le rappeler, ni exemptes de tout biais, ni irréprochables en matière d'incitations au conformisme ; qu'on songe au sort réservé, par l'université française, à des auteurs tels que Léon Walras ou, plus près de nous, Gérard Debreu et Fernand Braudel... 42

Il ne saurait évidemment être question de proposer ici un régime d'évaluation "clés en mains", alternatif au REQ. Tout au plus peut-on esquisser quelques pistes de réflexion, s'appuyant, certes, sur la réaffirmation de certains principes traditionnels, mais s'efforçant de ne pas trop succomber à l'illusion du retour à un âge d'or qui n'a jamais existé. Ces esquisses

\footnotetext{
${ }^{41} 1520$ citations de 629 auteurs pour Cédric Villani, et 102 citations de 52 auteurs pour Ngô Báo Châu

${ }^{42}$ Les deux premiers contraints à l'exil, le troisième "relégué" à l'École des Hautes Études.
} 
s'inscrivent dans la perspective tracée par l'Association Française d'Économie Politique, dans la mesure où elles s'appuient sur la conviction que la promotion systématique du pluralisme et de la diversité, en particulier sur les plans méthodologique et instrumental, est un des moyens privilégiés de lutte contre la sclérose et l'infertilité de la pensée économique contemporaine.

Enrayer la dérive quantitativiste du régime d'évaluation suppose, en premier lieu, que la substance du pouvoir d'évaluation ne soit plus accaparée par les revues et les listes, mais reprise par les instances qui en sont officiellement dotées. Or, une telle ré-appropriation ne peut être envisagée que si les instances prévues à cet effet en reviennent à une évaluation sur travaux (et non sur indicateurs). La dérive quantitativiste ne saurait être combattue sans que soit systématiquement réaffirmé le principe selon lequel toute évaluation sans lecture et appréciation des travaux devrait être proscrite.

De ce principe élémentaire découlent, concrètement, de multiples effets ou conséquences. La lecture et l'appréciation des travaux (ou d'une sélection de travaux) permettraient, tout d'abord, de rendre leur importance aux formats autres que l'article, laissant ainsi plus de place à des démarches moins "standard". Parallèlement, récuser toute évaluation sans lecture des travaux imposerait, matériellement, une réduction significative du nombre et de la fréquence des évaluations, laquelle favoriserait la qualité effective de l'évaluation, ${ }^{43}$ et limiterait, au moins à court terme, les incitations à la sur-publication. Enfin, l'application de ce principe permettrait, selon les préconisations de l'Académie des Sciences [Académie des Sciences 2011], d'interdire le recours aux indicateurs bibliométriques pour l'évaluation des jeunes chercheurs, et d'en circonscrire étroitement l'usage, pour les autres.

Le principe de lecture et d'appréciation des travaux induit, inévitablement, celui d'évaluation par les pairs. Or, le passé de l'université française a abondamment montré que l'évaluation par les pairs pouvait être, elle aussi, profondément conformiste et génératrice de biais. Il importe donc de ne pas se contenter d'un simple retour au principe d'évaluation par les pairs, mais d'organiser et de promouvoir systématiquement la diversité et le pluralisme, tant des instances qu'au sein des instances.

Selon la même logique, les modalités de fonctionnement et de délibération des instances d'évaluation devraient être élaborées et adaptées dans le souci permanent de valoriser le pluralisme, de mieux prendre en compte les recherches orphelines et/ou hétérodoxes, et de réfréner le conformisme "naturel" des chercheurs. Parallèlement, la logique d'examen devrait être systématiquement privilégiée, la logique de concours n'intervenant qu'en second temps, et seulement dans les cas où elle se révèle indispensable.

Pour soutenir aujourd'hui les progrès de la connaissance scientifique, et spécifiquement pour œuvrer au renouvellement de l'économie, il convient enfin de mener le combat du vocabulaire, beaucoup moins anodin qu'il n'y paraît. Face aux impasses de l'évaluation quantitative de la productivité académique, il importe de promouvoir, inlassablement, une logique d'appréciation de la contribution scientifique des chercheurs et des laboratoires.

Serge Walery, Aix-Marseille Université, 2011

\footnotetext{
${ }^{43}$ À cet égard, le modèle nord-américain peut être une source d'inspiration ; chaque universitaire y est évalué deux fois au cours de sa carrière (tenure, full-professor), mais de façon extrêmement approfondie. [Foucault, Lépinard, Lepinay, Mallard, 2007]
} 


\section{Références bibliographiques}

- M. Allais (2009). “C'est seulement dans la voie d'un immense effort de synthèse que les sciences sociales peuvent réaliser de grands progrès". L'Économie Politique, juillet 2009, $\mathrm{n}^{\circ}$ 43, pp 100-112.

- J. Apesteguia, I. Palacios-Huerta (2010). "Psychological pressure in competitive environments ; evidence from a randomized natural experiment." American Economic Review. Déc 2010, vol 100, issue 5, pp 2548-2564.

- F. Audier (2009). "L'évaluation et les listes de revues". La vie des idées.fr, 15 septembre 2009.

- J-C. Barbier, N. Matyjasik (2010). "Évaluation des politiques publiques et quantification en France : des relations ambiguës et contradictoires entre disciplines". Revue Française de Socio-Économie, $\mathrm{n}^{\circ}$ 5, $1^{\mathrm{er}}$ semestre 2010, pp 123-140.

- O. Beaumais (2007). “À propos de l'inscription sur la liste de qualification aux fonctions de maître de conférences. Une modélisation des décisions de la section sciences économiques du CNU". Revue économique, 2007-5, vol 58, pp 1111-1125.

- C. Bosquet, P-P. Combes, L. Linnemer (2010). "La publication d'articles de recherche en économie en France en 2008 ; disparités actuelles et évolutions depuis 1998." Rapport pour la Direction Générale de la Recherche et de l'Innovation (Ministère de l'Enseignement Supérieur et de la Recherche), version du $1^{\mathrm{er}}$ mars 2010.

- D. Card, G. Dahl (2011). "Family violence and football : the effect of unexpected emotional cues on violent behaviour." The Quarterly Journal of Economic,. (2011) CXXVI (1), pp 103143.

- P-P. Combes, L. Linnemer (2001). "La publication d'articles de recherche en économie en France." Annales d'Économie et de Statistique, n 62, 2001, pp 5-47.

- L. Cordonnier (2011). "Pourquoi les économistes se sont-ils trompés à ce point". L'Économie Politique, ${ }^{\circ}$ 50, avril 2011, pp 32-44.

- A. Desrosières (2010). La politique des grands nombres; histoire de la raison statistique. La Découverte, Paris, $3^{\text {ème }}$ édition, 2010 (1993).

- L. Egghe (2006). "Theory and practise of the g-index." Scientometrics, vol 69, n 1(2006), pp 131-152.

- M. Foucault, E. Lépinard, V. Lepinay, G. Mallard, (2009). "Pour des université plus justes". La vie des idées.fr, 17 février 2009.

- Y. Gingras, (2008). "Du mauvais usage de faux indicateurs". Revue d'Histoire Moderne et Contemporaine, 2008/5, $\mathrm{n}^{\circ}$ 55-4 bis, pp 67-79. 
- Hirsch, J.E., (2005), “An index to quantify an individual's research output”, Proceedings of the National Academy of Sciences, 102(46), 16569-16572.

- G. Hodgson, H. Rothman (1999). "The editors and authors of economics journals : a case of institutional oligopoly?" Economic Journal, February 1999, pp 165-186.

- P. Jeannin (2004). "Les économistes et leurs revues." Revue d'Économie Politique, 114(3), mai-juin 2004, pp 275-288.

- S. D. Levitt, S. J. Dubner (2005). Freakonomics, William Morrow, New-York.

- F. Nietzsche (1874). Considérations inactuelles 3 ; Schopenhauer éducateur. Gallimard, Folio, Paris, 1992.

- C. N. McKinney, N. G. Rupp (2002). "The publication patterns of the elite economics departments : 1995-2000.” Eastern Economic Journal, Vol 28, n 4, 2002, pp 523-538.

- A. Ogien (2010). "La valeur sociale du chiffre ; la quantification de l'action publique entre performance et démocratie." Revue Française de Socio-Économie, n ${ }^{\circ} 5,1^{\mathrm{er}}$ semestre 2010, pp $19-40$.

- B. Perret (2006). "De l'échec de la rationalisation des choix budgétaires (RCB) à la loi organique relative aux lois de finances (LOLF)." Revue française d'administration publique, $\mathrm{n}^{\circ} 117,2006$, pp 31-41.

- B. Perret (2008). L'évaluation des politiques publiques. La Découverte, Repères, Paris.

- D. Pontille, D. Torny (2010). "Revues qui comptent, revues qu'on compte : produire des classements en économie et gestion." Revue de la Régulation, capitalisme, institutions, pouvoirs, $\mathrm{n}^{\circ} 8,2^{\mathrm{ème}}$ semestre 2010.

- N. Postel (2011). “Le pluralisme est mort, vive le pluralisme !" L'Économie Politique, n 50, avril 2011, pp 6-31.

- G. Raveaud (2011). "Un enseignement pluraliste des représentations de l'économie". L'Économie Politique, ${ }^{\circ}$ 50, avril 2011, pp 59-70.

- G. Ripka (2002). Vivre savant sous le communisme, Belin, collection Débats, Paris.

- R. J. \& V. M. Schiller (2011). "Economists as worldly philosophers." Yale University, Cowles Foundation, Discussion Paper n ${ }^{\circ}$ 1788, mars 2011.

- « Du bon usage de la bibliométrie pour l'évaluation individuelle des chercheurs. » Institut de France, Académie des Sciences. Rapport remis à Madame la Ministre de l'Enseignement Supérieur et de la Recherche. 17 janvier 2011.

- «L" "excellence scientifique" comme prétexte au scientisme», lettre ouverte par des membres élus de la section Économie du CNU, 21 janvier 2010. 